\title{
Erratum: Magnetotransport signatures of three-dimensional topological insulator nanostructures [Phys. Rev. B 97, 245429 (2018)]
}

\author{
Kristof Moors $\odot$, Peter Schüffelgen, Daniel Rosenbach, Tobias Schmitt, Thomas Schäpers, and Thomas L. Schmidt
}

(Received 8 February 2021; published 22 February 2021)

DOI: 10.1103/PhysRevB.103.079902

The in-plane orientation angles of the kink structure $\left(\gamma_{K}\right)$ and the magnetic field $\left(\gamma_{B}\right)$ were oppositely defined in the simulations of the right-angle kink that are presented in Fig. 5. Consequently, the statements in the text regarding the transmission across the right-angle kink for the aligned and transverse orientations of the magnetic field are inverted, as well as the schematic depiction in Figs. 8(a) and 8(b). Opposite to what is stated, the transmission across the right-angle kink is perfect for the transverse orientation and highly reduced for the aligned orientation.

We also identified four typographical errors in the paper. First, equation (8) should read:

$$
\begin{aligned}
\mathcal{H}^{3 \mathrm{D}}(\mathbf{k}) & \equiv \epsilon(\mathbf{k})+\tau_{z} M(\mathbf{k})+\tau_{x} A_{\perp}\left(\sigma_{x} k_{x}+\sigma_{y} k_{y}\right)+\sigma_{z} \tau_{x} A_{z} k_{z}, \\
\epsilon(\mathbf{k}) & \equiv C_{0}-C_{\perp}\left(k_{x}^{2}+k_{y}^{2}\right)-C_{z} k_{z}^{2}, \quad M(\mathbf{k}) \equiv M_{0}-M_{\perp}\left(k_{x}^{2}+k_{y}^{2}\right)-M_{z} k_{z}^{2} .
\end{aligned}
$$

Second, the value of $M_{z}$ of the $\mathrm{Bi}_{2} \mathrm{Se}_{3}$ (B) parameter set in Table I should read $M_{z}=6.86 \mathrm{eV} \AA^{2}$. Third, the magnitudes of the applied magnetic fields that are mentioned in Secs. IV B and IV C, and the captions of Figs. 5 and 6, are presented assuming $\Phi_{0} \equiv h /(2 e)$, whereas $\Phi_{0} \equiv h / e$ is assumed throughout the remainder of the text. Hence, the mentioned magnitudes should be divided by a factor of two for consistency. All the magnitudes are chosen such that a gap closing is realized in the wire legs (the half-integer flux-pierced regime) when the external magnetic field and the leg have a relative angle of $\pi / 6, \pi / 4$, or $\pi / 3$. Fourth, the legends in Figs. 7(a) and 7(b) should read $B_{\perp} / B_{\|}=0, B_{\perp} / B_{\|}=\tan (\pi / 6)$, and $B_{\perp} / B_{\|}=\tan (\pi / 3)$, respectively.

All the corrected figures are presented in Fig. 1. The other parts of the text, figures, and conclusions remain unchanged. 


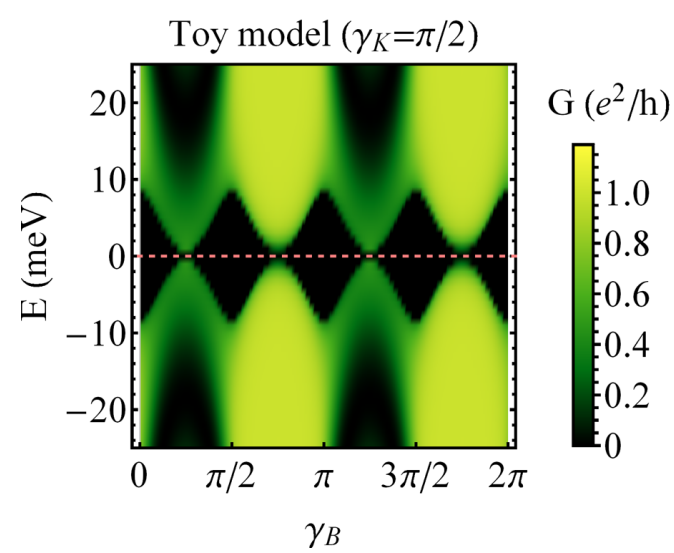

(a)

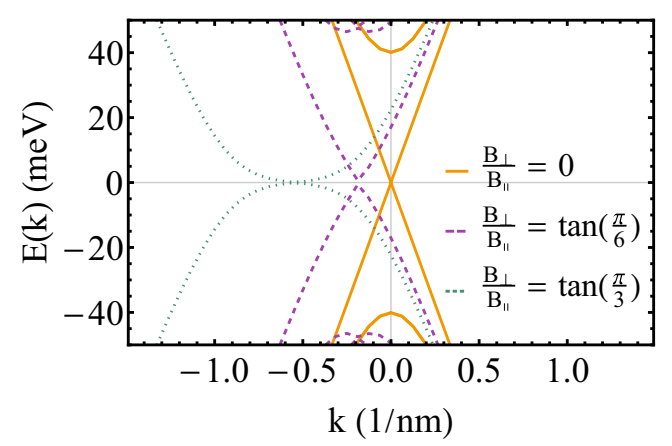

(c)

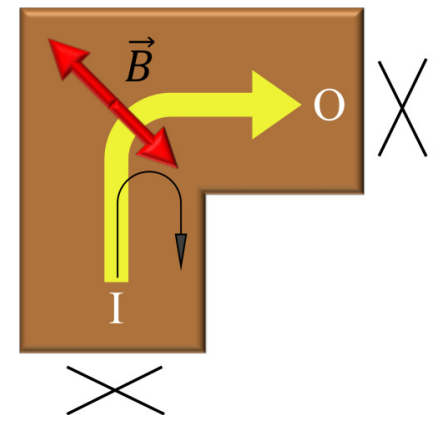

(e)

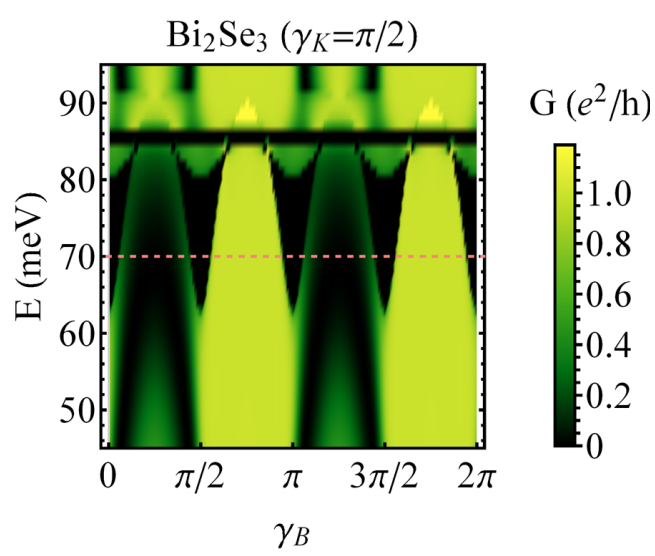

(b)

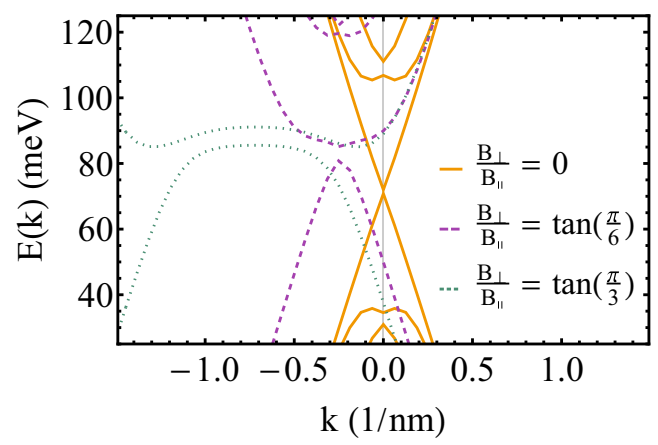

(d)

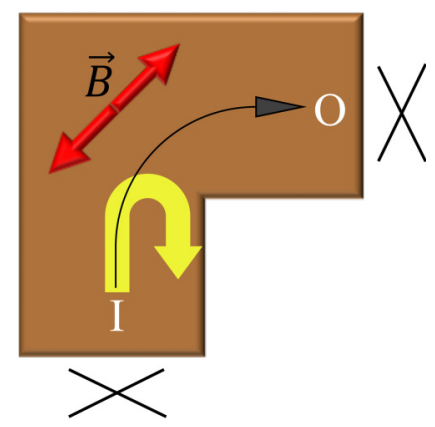

(f)

FIG. 1. (a),(b) Corrected versions of Figs. 5(a) and 5(b). (c),(d) Corrected versions of Figs. 7(a) and 7(b). (e),(f) Corrected versions of Figs. 8(a) and 8(b). 